\title{
Hernia Pulmonar Secundaria a la Colocación de Tubo de Tórax en Paciente con Hemotórax Traumático
}

\author{
${ }^{1}$ Morris Sasson, ${ }^{2}$ Rafael Andrade-Alegre
}

\section{RESUMEN}

La mayoría de las hernias pulmonares son congénitas, traumáticas o post-quirúrgicas. Hay muy pocos casos reportados secundarios a la colocación de tubo de tórax. Presentamos el caso de una hernia pulmonar luego del retiro del tubo de tórax en un paciente con hemotórax secundario a trauma penetrante.

Palabras clave: Tubo de tórax, Hernia pulmonar, Trauma.

How to cite this article: Sasson M, Andrade-Alegre R. Hernia Pulmonar Secundaria a la Colocación de Tubo de Tórax en Paciente con Hemotórax Traumático. Panam J Trauma Crit Care Emerg Surg 2014;3(2):82-84.

Source of support: Nil

Conflict of interest: None

\section{ABSTRACT}

Most cases of lung herniation are either congenital, secondary to trauma or surgery. Very few cases have been reported secondary to the insertion of chest tubes. We present a case of lung hernia after the removal of a chest tube that was inserted to treat an hemothorax secondary to penetrating trauma.

Keywords: Chest tube, Lung hernia, Trauma.

\section{INTRODUCTION}

El primer informe de hernia pulmonar corresponde a Roland en 1499 (citado en). ${ }^{1}$ En 1845 Morel-Lavallée propuso la clasificación de hernias pulmonares más aceptada hasta el día de hoy. Esta clasificación se basa en localización anatómica y la etiología. La clasificación etiológica divide las hernias en congénitas y adquiridas. Estas últimas representan el $80 \%$ de los casos. La mayoría son secundarias a trauma o cirugía. $^{2}$ Lo y Shapiro ${ }^{3}$ encontraron en la literatura médica de habla inglesa unos 400 casos hasta el 2010. Las hernias pulmonares asociadas a colocación de tubos de tórax son aún más raras. ${ }^{2}$

\footnotetext{
${ }^{1}$ Intern, ${ }^{2}$ Chief Thoracic Surgery

1,2Department of Surgery, Santo Tomas Hospital, Panamá Panama

Corresponding Author: Rafael Andrade-Alegre, Chief Thoracic Surgery, Department of Surgery, Santo Tomás Hospital, Panamá, Panama, Phone: (507)507 5600 e-mail: toravasc@cwpanama.net
}

\section{Reporte de Caso}

Un hombre de 52 años sufrió una herida por proyectil de arma de fuego en el tórax posterior izquierdo, desarrollando hemotórax para lo que se le colocó un tubo de tórax. Una vez resuelto el hemotórax, el tubo de tórax fue retirado y el paciente dado de alta con control ambulatorio. Dos semanas después, el paciente regresa al hospital quejándose de tos no productiva, dolor torácico y dificultad respiratoria. El paciente se encontraba afebril y hemodinámicamente estable. Al exámen físico se encontró un discreto abultamiento en el área de la toracostomía sin signos inflamatorios (Fig. 1) y a la auscultación pulmonar los ruidos respiratorios estaban disminuídos en la base posterior izquierda. También se auscultaban algunos estertores. No se evidenciaron otras alteraciones en la evaluación física. La biometría hemática reveló una cuenta de glóbulos blancos de 20,800 por mm. ${ }^{3}$ y la radiografía de tórax PA mostró una opacidad redondeada hacia la base pulmonar izquierda. Seguídamente se realizó tomografía computada del tórax que evidenció una lesión de $12.5 \times 8.2 \times 5.5 \mathrm{~cm}$. En la mitad inferior del tórax izquierdo (Fig. 2A). Se demostró refuerzo de la periferia al administrar medio de contraste. También fue evidente pequeños niveles aire-líquido y fragmentos del proyectil. Un hallazgo inesperado fue la aparición de protrusión de parénquima pulmonar a través de la pared torácica en el tercio medio del tórax izquierdo (Fig. 2B).

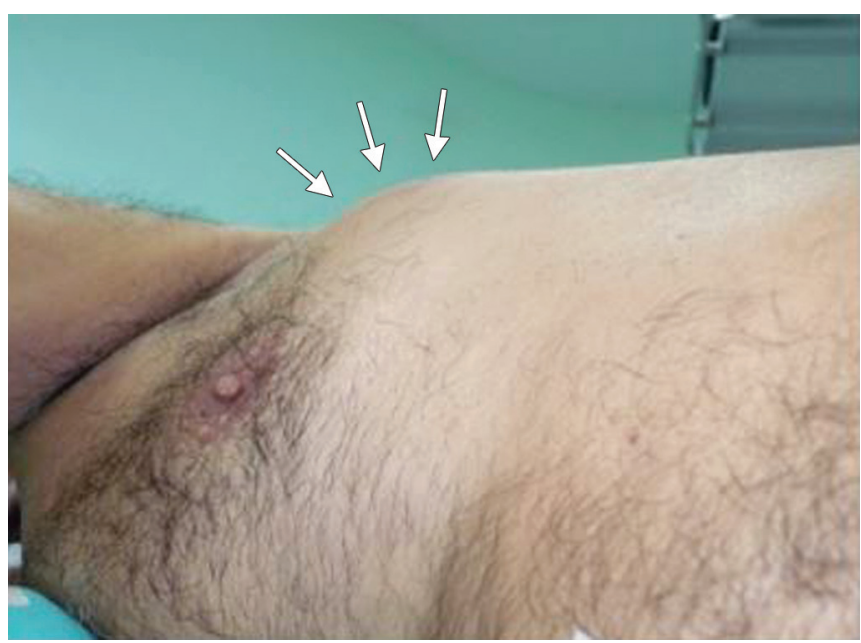

Fig. 1: Paciente en decúbito lateral derecho. Las flechas señalan e discreto aumento de volumen en el área de la toracostomía cerrada 

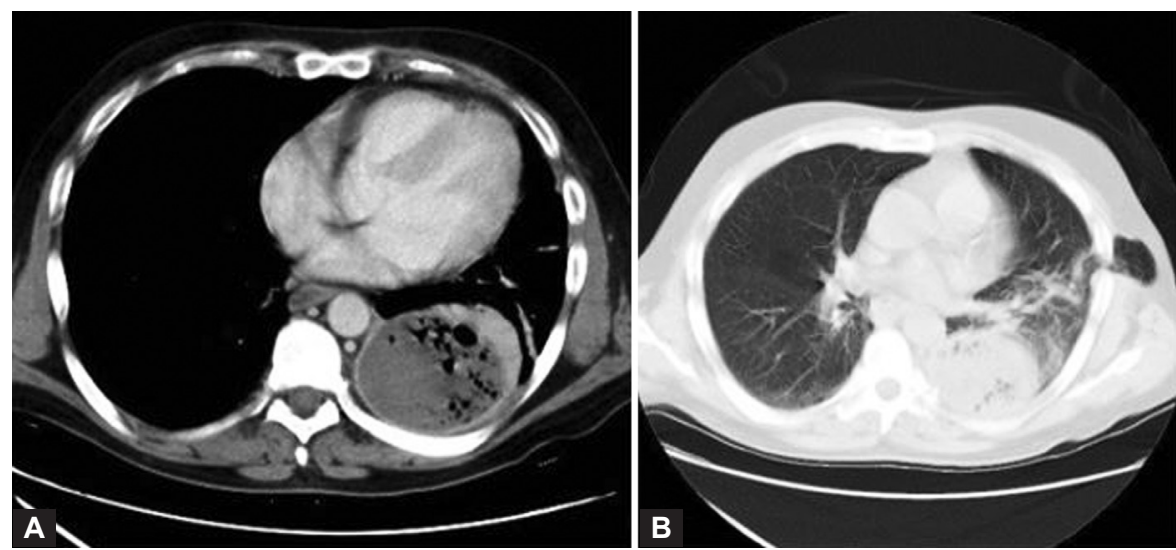

Figs 2A and B: Tomografía computada del tórax: (A) Se aprecia colección loculada posterior basal izquierda con refuerzo periférico y aire en su interior, (B) Ventana pulmonar que muestra protrusión de tejido pulmonar a través de la pared torácica

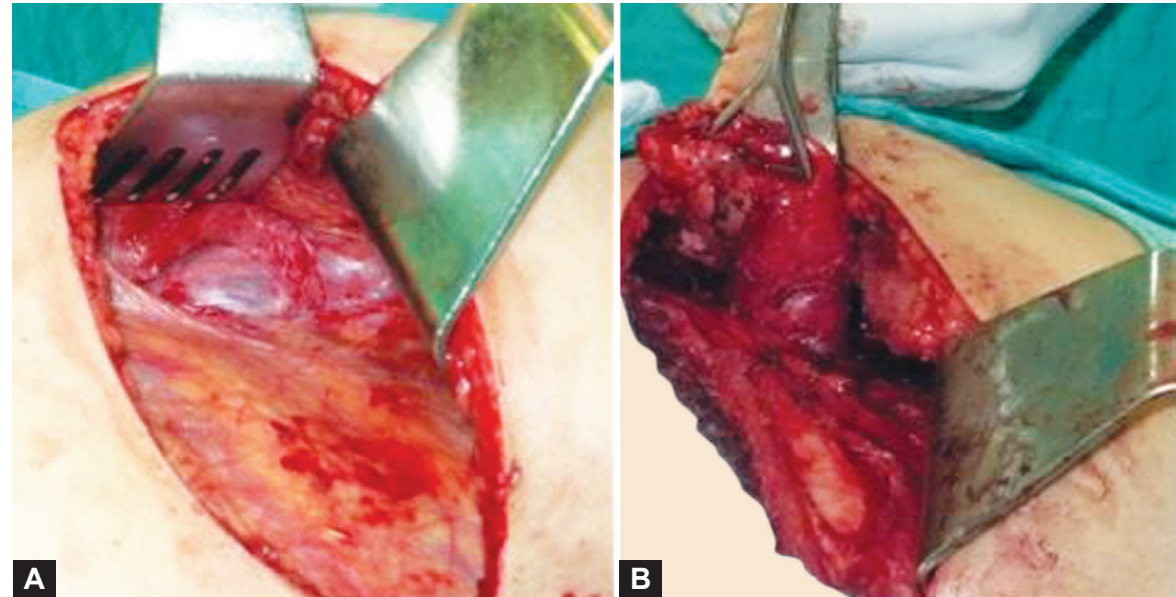

Figs 3A and B: Hernia pulmonar: (A) Incisión torácica izquierda donde se aprecia tejido pulmonar con adherencias,

(B) Disección del parénquima pulmonar viable previo a su reducción

Con estos hallazgos se hizo el diagnóstico de hemotórax coagulado y hernia pulmonar intercostal. El paciente fue llevado al salón de operaciones y se realizó toracotomía lateral a nivel del $6^{\circ}$ espacio intercostal. Se apreció la língula protruyendo a través de un defecto intercostal de aproximadamente $4 \mathrm{~cm}$ (Fig. 3A). El tejido pulmonar era viable (Fig. 3B), así que la hernia es reducida y el defecto intercostal en forma de anillo es reparado con puntos pericostales. Luego se evacúa el hemotórax y se realiza decorticación pulmonar. El paciente tuvo un postoperatorio sin complicaciones y fue dado de alta al $6^{\circ}$ día. En seguimiento a 12 meses no ha habido recurrencia.

\section{DISCUSIÓN}

Las hernias pulmonares son asintomáticas en la mayoría de los casos. El diagnóstico con frecuencia se hace de forma incidental. En otros casos, la herniación pulmonar puede presentarse con síntomas y signos muy sutiles. Los pacientes sintomáticos pueden tener aumento de volumen en la pared torácica, el que puede crepitar o darse una sensación esponjosa a la palpación. ${ }^{4}$ En raras ocasiones, el paciente puede presentarse con masa dolorosa palpable debido a incarceración o estrangulación. Nuestro paciente regresó al hospital por manifestaciones clínicas asociadas al hemotórax coagulado. No presentaba dolor en el área de la herida del tubo de tórax y el aumento de volumen en la pared era muy tenue. Las hernias pulmonares pueden hacerse evidentes cuando el paciente hace maniobra de Valsalva. ${ }^{5}$

La radiografía de tórax PA usualmente no es útil para hacer el diagnóstico de hernia pulmonar, como ocurrió en nuestro caso. Las radiografías laterales u oblícuas del tórax pueden demostrar tejido pulmonar fuera de la caja torácica, ${ }^{6}$ sin embargo, la tomografía computada del tórax es el estudio de elección para demostrar una hernia pulmonar. También provee información importante en relación a la pared torácica, espacio pleural, localización exacta de la hernia y tamaño del defecto. Todos éstos, aspectos cruciales en la planeación quirúrgica. ${ }^{7,8}$

El tratamiento de la hernia pulmonar es todavía controversial. ${ }^{9,10}$ Ambos manejos conservador o quirúrgico han sido recomendados. Las hernias asintomáticas particularmente aquellas en región supraclavicular o debajo de la escápula, pueden no requerir tratamiento quirúrgico. 
El aumento de tamaño, dolor o sospecha de incarceración son indicaciones de cirugía. Los defectos pequeños pueden aumentar el riesgo de incarceración por lo que en estos casos la cirugía no debe ser retrasada. ${ }^{11}$

El manejo quirúrgico de las hernias pulmonares implica liberación de adherencias, reducción del tejido pulmonar y cierre del defecto herniario. No hay una técnica para todos los pacientes, de tal forma que el tratamiento debe ser individualizado. Existen diferentes técnicas quirúrgicas para reparar estas hernias: fijación pericostal, uso de material protésico, tejido autólogo y flaps musculares complejos. ${ }^{12,13}$ En nuestro caso elegimos proceder con fijación pericostal dado que el defecto era pequeño y la aproximación intercostal se hizo fácilmente.

En resumen, este es un caso muy raro de hernia pulmonar asociado a la colocación de tubo de tórax. Es importante tener un alto índice de sospecha. La tomografía computada es modalidad diagnóstica de elección.

\section{REFERENCIAS}

1. Montgomery JG, Lutz H. Hernia of the lung. Ann Surg 1925;82:220-231.

2. Moncada R, Aruna V, Gimenez C, et al. Congenital and acquired lung hernias. J Thorac Imaging 1996;11:75-82.

3. Lo D, Shapiro M. Extrathoracic lung herniation from blunt trauma. A review. J Surg Radiol 2010;1:20-30.
4. Khalil MW, Masala N, Waller DA, Cardillo G. Surgical repair of post-traumatic lung hernia using video-assisted open technique. Interact Cardiovasc Thorac Surg 2008;7:506-507.

5. DiMarco AF, Oca O, Renston JP. Lung herniation. A cause of chronic chest pain following thoracostomy. Chest 1995 Mar; 107(3):877-879.

6. Sadler MA, Shapiro RS, Wagreich J, et al. CT diagnosis of acquired intercostal lung herniation. Clinical Imaging 1997;21:104-106.

7. Tamburro F, Grassi R, Romano S, Del Vecchio W. Acquired spontaneous intercostal hernia of the lung diagnosed on helical CT. AJR 2000;174:876-877.

8. Athanassiadi K, Bagaev E, Simon A, Haverich A. Lung herniation: a rare complication in minimally invasive cardiothoracic surgery. Eur J Cardiothorac Surg 2008;33:774-746.

9. Emberger JS, Racine L, Maheshwari V. Lung hernia associated with hemothorax following cardiopulmonary resuscitation. Respir Care 2011;56(7):1037-1039.

10. Bikhchandani J, Balters MW, Sugimoto JT. Conservative management of traumatic lung hernia. Ann Thorac Surg 2012;93(3):992-994.

11. Weissberg D, Refaely Y. Hernia of the lung. Ann Thorac Surg 2002;74:1963-1966.

12. Wiens S, Hunt I, Mahood J, Valji A, Stewart K, Bédard EL. Novel fixation technique for the surgical repair of lung hernias. Ann Thorac Surg 2009 Sep;88(3):1034-1035.

13. Lozano-Corona R, Loyola-García, Partida IC, RodriguezOrtega F. Successful treatment of flail chest with condrosternal disruption and traumatic parasternal lung hernia with titanium rib bridges. BMJ Case Reports Published 22 April 2013. 